\title{
Recherches sur le pigment rouge des poissons d'eau douce
}

par

\section{Emile ANDRÉ}

Dans un précédent travail ${ }^{1}$ nous avons démontré que le pigment rouge des Truites fario est une carotine et qu'il a pour origine les Crustacés ingérés comme aliments; les expériences qui ont conduit à ces notions, nous les avons reprises en les faisant porter sur d'autres espèces de Poissons appartenant aux familles des Salmonides, des Acanthopsides, Cyprinides et Cottides et nous avons également fait quelques observations sur la pigmentation des Corégones, Brochets, etc. Nos expériences consistaient donc à élever deux lots de diverses espèces de poissons, l'un des lots étant nourri exclusivement de Gammarus, le deuxième, de vers de terre hâchés ou d'Enchytraeus albidus. Dans les lignes qui suivent, nous emploierons pour abréger les expressions "lot-Gammarus " et "lot-vers".

Chez toutes les espèces de poissons examinées, à l'exception des Brochets, nous avons constaté dans la peau la présence d'une carotine, cela en faisant les deux réactions suivantes:

1) les cellules pigmentaires jaunes ou rouges, fraîches, étaient traitées par l'acide sulfurique; celui-ci a toujours provoqué le verdissement ou le bleuissement du pigment;

2) les parties pigmentées de la peau, en général les nageoires, étaient desséchées dans l'exsiccateur, puis traitées par le chloroforme; la solution obtenue était additionnée d'acide sulfurique qui la virait au bleu et au vert, principalement dans le voisinage de la surface de contact des deux liquides.

Les résultat de ces deux réactions ont toujours été positifs, mais il faut faire remarquer que dans la première le virage du pigment

- $1 \mathrm{E}$. André. Influence de l'alimentation sur la pigmentation cutanée des Salmonides. Revue suisse de Zoologie, vol. 33, p. 659. 1926.

Rev. Suisse de Zool. T. 35. 1928. 
se manifestait tantôt instantanément, tantôt au bout d'un temps plus ou moins long, jusqu'à quelques heures. Il faut en conclure, ou bien qu'il y a plusieurs espèces de carotines, ou bien que l'action de l'acide sulfurique est plus ou moins rapide, peut-être suivant la quantité de graisse associée à la carotine. Inutile de dire que le bleuissement varie en intensité suivant la quantité de carotine contenue dans les cellules et que ce sont les cellules rouge vif qui donnent la coloration bleue la plus forte, tandis que les cellules jaunes ne montrent qu'un léger verdissement. Chez certains Poissons, nous n'avons trouvé que des cellules jaunes; faut-il en conclure qu'il y a une carotine jaune et une carotine rouge? Cela confirmerait les données de Maly et de Vegezzi ${ }^{1}$, qui ont l'un et l'autre travaillé sur les œufs de Maia; de plus les mesures spectroscopiques que nous avons faites, et que nous comptons reprendre plus tard, sembleraient en faveur de cette hypothèse. D'autre part, nous avons vu chez les alevins de Salmo fontinalis du lot-Gammarus les taches de la peau, d'abord jaune très pâle, passer ensuite au jaune vif, au rouge orange et enfin au rouge vif; en outre chez des individus de plusieurs espèces de Poissons nous avons observé toutes les transitions entre les cellules jaunes et les cellules rouges. De ces faits on pourrait alors induire que, s'il y a deux carotines, une jaune et une rouge, la première serait l'état initial de la seconde.

Avant de donner les résultats de nos élevages et d'en tirer quelques conclusions, nous devons reprendre trois points que nous touchions dans notre précédent travail.

Comme nous l'avons déjà mentionné et comme on le verra plus loin, les carotinoïdes se fixent rapidement dans les téguments; mais ce dépôt est-il définitif ou temporaire? Pour répondre à cette question, nous avons élevé en aquarium des Salmo fario en les nourissant exclusivement d'asticots et de vers de terre; les trois individus en expérience étaient porteurs de points rouges, pas très vifs cependant. Une des Truites (poids 195 gr.) est morte après 61 jours, une deuxième (poids 110 gr.) après 128 jours et la troisième (poids 310 gr.) a été sacrifiée au bout de 250 jours. Chez aucun de ces poissons les taches rouges n'avaient pâli de notable

1 R. Maly. Ueber die Dotterpigmente. Monatshefte für Chemie, vol. 2, p. 351-362. 1881.

G. VEGEZzi. Recherches sur quelques pigments des Invertébrés. Inaug. diss., Fribourg. 1916. 
façon; on peut donc en conclure que le pigment rouge fixé dans les téguments, des adultes tout au moins, présente une certaine stabilité. Nous disons "chez les adultes", car il est probable que, si l'expérience avait porté sur des sommerlings ou des jahrlings, les résultats en eussent été différents. Il ne semblent guère admissible en effet que la faible quantité de pigment rouge fixée par les jeunes puissent suffirent à les colorer jusqu'à l'âge adulte.

Dans le travail cité plus haut, nous émettions l'idée que certains Entomostracés porteurs de lipochromes rouges pouvaient aussi céder ceux-ci à des poissons et contribuer à les pigmenter en rouge. Cette supposition, toute vraisemblable qu'elle soit, devait être confirmée par l'expérience, mais dans un laboratoire il n'est pas possible d'avoir, comme dans un établissement de pisciculture, des élevages de Daphnies suffisamment productifs pour alimenter des poissons pendant quelque temps. Cependant nous pouvons donner une réponse affirmative à la question que nous posions en nous appuyant sur deux faits.

Le Bettmersee, petit lac des Alpes valaisannes, situé à 2050 mètres d'altitude, est peuplé de nombreux Vairons (Phoxinus phoxinus); ceux-ci au mois d'août 1927 montraient une riche coloration rouge, principalement sur les nageoires et sur la partie antérieure de la face centrale. Or, dans ce lac nous avons vainement cherché des Gammarus, tandis que les Entomostracés planctoniques y abondaient. Il semble donc légitime d'attribuer à ces derniers les dépôts de carotinoïdes de la peau des Vairons.

D'autre part, grâce à la complaisance de M. M. VougA, inspecteur général de la Pêche à Neuchâtel, nous avons pu examiner une vingtaine de jeunes Salmo fontinalis, longs de 7 à 9 cent., élevés dans des aquariums de l'établissement piscicole du Pervou et ayant été alimentés uniquement de Daphnies (Daphnia pulex). Tous les individus avaient les nageoires colorées comme suit : nageoires pectorales, cellules rouges surtout dans la région antérieure;

nageoires abdominales, cellules rouges, assez abondantes, surtout vers le bord antérieur;

nageoire dorsale, quelques cellules rouges et quelques cellules jaunes, c'est la nageoire la moins colorée;

nageoire adipeuse, quelques cellules rouges, surtout vers le bord antérieur; 
nageoire anale, cellules rouges assez abondantes, surtout dans la région antérieure;

nageoire caudale, cellules rouges, surtout vers les bords supérieur et inférieur.

De plus, les flancs présentaient des taches, allant du jaune pâle au rouge vif; d'une façon générale on peut dire que le nombre et l'intensité de la coloration de ces taches augmentait avec la taille. De cette observation, on peut conclure que pour les Salmo fontinalis, comme pour les Truites fario ${ }^{1}$ les carotinoïdes se fixent d'abord sur les nageoires, puis dans la peau des flancs; là apparaissent d'abord des taches jaunes qui passent ensuite au rouge, le phénomène commençant par les taches situées au-dessus de la ligne latérale. Le pigment des nageoires montrait la réaction caractéristique avec l'acide sulfurique. La chair n'était pas saumonée, même après coction. La graisse était incolore. A cela, il faut ajouter que le pigment rouge des Daphnia pulex en solution chloroformique bleuit par l'acide sulfurique.

Nous avions, en outre, posé cette question: puisqu'il y a identité entre le pigment rouge de la peau et celui de la chair saumonée, pourquoi toutes les truites à points rouges ne sont-elles pas saumonées? Et nous y avions répondu par la supposition que les carotines ne se fixent dans la musculature que lorsque la peau et les ovaires en sont pour ainsi dire saturés ${ }^{2}$. En réalité la question est plus complexe que nous ne le pensions et notre explication paraît insuffisante. En effet, nous avons eu l'occasion d'examiner 6 Truites fario de la Reuss, de 150 à 350 grammes et chez tous ces individus la chair était intensément saumonée, tandis que les points rouges de la peau étaient fort pâles. Nous avons également noté que chez ces Truites les muscles basilaires de la nageoire dorsale étaient restés incolores et tranchaient de façon très nette sur le reste de la musculature; ce fait qui, à notre connaissance, n'a pas encore été signalé, est peut-être dû à une plus faible teneur en graisse des muscles restés incolores.

Nous donnerons maintenant les résultats de nos expériences qui ont porté sur 12 espèces de poissons.

1 André, loc. cit.

2 André, loc. cit 
Truite arc-en-ciel (Salmo irideus Gibb.). Tous les alevins de cette espèce ${ }^{1}$ que nous destinions à nos expériences sont morts avant la résorption de la vésicule vitelline; mais nous avons pu examiner deux individus de 6 à $7 \mathrm{~cm}$., provenant de l'établissement piscicole du Pervou (Neuchâtel) et nourris jusqu'alors exclusivement de pulpe de rate; ni l'un, ni l'autre ne possédait des érythrocytes.

Chez les adultes, provenant de Grémaz, nous avons constaté que le pigment, bien qu'il soit carminé et non pas rouge, est aussi une carotine; il se trouve dans la région operculaire, sur les flancs et dans les nageoires.

Relativement à la "saumonisation " de la chair, nous avons noté qu'un individu d'environ 125 gr. montrait, après 76 jours d'alimentation en Gammarus, la musculature légèrement colorée, tandis qu'un autre individu de même taille et nourri de la même façon pendant 545 jours, n'était pas saumoné; mais il est utile de dire qu'à ce dernier les Gammarus n'ont été donnés que d'une manière un peu parcimonieuse pour sa taille.

Truite are-en-ciel var. albinos. Pour nos expériences ces Truites constituent un matériel très précieux, puisque l'absence de mélanine, pigment qui dans beaucoup de cas masque les carotines, permet d'observer la première apparition de celles-ci et de noter exactement les régions qui sont susceptibles de les fixer. Nous adressons ici tous nos remerciements à M. L. Kreitmann, inspecteur des Eaux et Forêts à Thonon (Haute-Savoie), qui a bien voulu nous donner quelques individus de cette intéressante variété; celle-ci est actuellement élevée en assez grand nombre dans les bassins de l'établissement de pisciculture de Thonon ${ }^{2}$. Avant l'élevage dans nos aquariums, les poissons, qui mesuraient

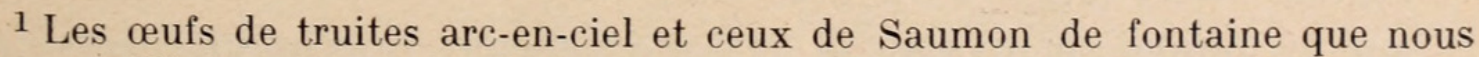
avons utilisés ont été aimablements mis à notre dispositions par M. BENoIT, propriétaire de l'établissement piscicole de Gremaz (Ain) auquel nous exprimons ici toute notre gratitude; nous adressons aussi nos plus vifs remerciements à M. M. VougA, inspecteur général de la pêche à Neuchâtel, qui a facilité nos recherches en nous envoyant de nombreux sujets et dont nous avons eu souvent l'occasion d'apprécier la compétence et le dévouement.

- 2 Voir: L. Kreitmann. Une nouvelle variété de Truite arc-en-ciel albinos. Bull. de la Soc. Centrale d'aquiculture et de pêche. Vol. 34, p. 24.1927. 
de 7 à $9 \mathrm{~cm}$., rı'étaient pas absolument incolores; ils montraient quelques cellules pigmentaires jaune-pâle, probablement parce qu'ils avaient rencontré quelques rares Gammarus dans les bassins de Thonon ou peut-être parce qu'ils provenaient d'œufs à vitellus pourvu de carotine ${ }^{1}$. Voici quelle était la répartition du pigment ${ }^{1}$ : nageoires pectorales, légèrement jaunes, surtout vers leur bord antérieur;

nageoires abdominales, incolores:

nageoire dorsale, très faiblement jaune, surtout dans la région du bord antérieur;

nageoire adipeuse, jaune très pâle dans la région basale;

nageoire anale, quelques cellules jaune-pâle, surtout dans la région basale antérieure;

nageoires caudales, faiblement jaune vers les bords supérieur et inférieur.

Pour toutes les nageoires, la coloration n'était pas uniforme; elle formait de petites taches, uniquement dans la membrane interradiaire.

Après un jour et demi les individus du lot-Gammarus sont très manifestement plus colorés que ceux qui sont alimentés de vers de terre et de viande hâchée. Au bout d'un laps de temps de 71 jours, les truitelles du lot-vers sont incolores, tandis que les autres sont richement colorées, à tel point qu'à distance on aurait pu les prendre pour des Orfes un peu pâles. Ils avaient prospéré beaucoup plus que leurs congénères du lot-vers (le plus grand mesurait 13,5 cm.), peut-être par le fait qu'ils avaient constamment des Gammarus à disposition, tandis que les autres n'étaient nourris que deux fois par jour. Voici comment était répartie la coloration rouge des truitelles du lot-Gammarus:

toutes les nageoires sont colorées, les nagoires abdominales, anale et caudale très vivement, les autres beaucoup moins;

région operculaire d'un rose vif;

sur les flancs au niveau de la ligne latérale, une bande carminée commençant à $1,5 \mathrm{~cm}$. en arrière des ouïes et se terminant à $2 \mathrm{~cm}$. en avant de la queue; corps rose doré, plus clair au-dessous de la ligne latérale, la face ventrale étant cependant incelore, à part quelques taches orangées en avant des nageoires abdominales;

1 Voir: ANDRÉ. loc. cit. 
chair nettement saumonée;

graisse des viscères, de même coloration, mais plus accentuée encore.

On sait que la lumière a une influence sur la formation de la mélanine ${ }^{1}$; en est-il de même pour la fixation des carotines? Pour répondre à cette question, nous avons élevé une truitelle du lotvers dans l'obscurité complète en laissant à sa disposition une copieuse provision de Gammarus; après quatre jours nous l'avons amenée à la lumière et constaté que le corps et les nageoires étaient déjà vivement colorés, moins cependant que chez les individus nourris de crevettes pendant 71 jours. Nous avons aussi tenté d'élever à la lumière, en la nourrissant de Gammarus, une truitelle du lot-vers aveuglée par la section des nerfs optiques; mais nous avons arrêté l'expérience au bout de 2 jours après avoir constaté que l'animal ne prenait aucun aliment; l'intestin était complètement vide.

Saumon de fontaine (Salmo fontinalis Mitch.). L'élevage a été prolongé pendant 60 jours après l'éclosion. Le lot-Gammarus a été nourri d'abord de ces Crustacés séchés et broyés, puis frais et broyés. Les alevins de ce lot ont peu prospéré tant qu'ils n'ont eu à leur disposition que les Gammarus sees et la coloration rouge visible à l'œil nu, n'a fait son apparition que vers le $35^{\text {me jour }}$ mais déjà au bout de 12 jours on pouvait reconnaître au microscope des cellules jaunes dans les nageoires caudale, dorsale et anale. A la fin de l'élevage soit donc aprés 60 jours, les nageoires abdominales, dorsale, caudale et anale montraient des plages rouges visibies à l'œil nu. Les nageoires pectorales et adipeuse étaient incolores, mais le microscope y révélait quelques rares cellules rouge-orange. Dans la peau, des cellules semblables étaient assez régulièrement distribuées. La chair était incolore et le restait après coction. Les alevins du second lot ne montraient pas trace de pigment rouge.

Chez un adulte de 125 gr., nourri de Gammarus pendant 76 jours la chair était saumonée; l'individu-témoin, de poids égal à peu près, nourri de vers de terre et de viande pendant le même laps de temps n'était pas saumoné.

- 1 Voir: P. Murisier. Le pigment mélanique de la Truite (Salmo lacustris) et le mécanisme de sa variation quantitative sous l'influence de la lumière. Rev. suisse de Zool., vol. 28. 1920-1921. 
Ombre (Thymallus thymallus L). Nous n'avons pas fait d'élevage de cette espèce mais nous avons pu faire quelques observations sur les érythrocytes d'un individu provenant de l'Areuse que nous devons encore à l'obligeance de M. Vouga. La répartition du pigment rouge ou jaune est la suivante:

nageoires pectorales, celiules jaunes peu abondantes, mais distribuées assez régulièrement sur toute l'étendue de la nageoire;

nageoires abdominales, grosses cellules rouge foncé, rondes ou ramifiées; quelques petites cellules jaunes; quelques grosses cellules bleues;

nageoire dorsale, cellules rouge foncé, commme ci-dessus, surtout abondantes dans les ters antérieur et postérieur;

nageoire adipeuse, pas d'érythrocytes;

nageoire anale, quelques très rares cellules rouges comme ci-dessus et quelques cellules jaunes très rares aussi;

nageoire caudale, pas d'érythrocytes.

Le pigment rouge du Thymallus est d'une nature autre que celui des Poissons examinés (à part le Spirlin); il est d'un rouge pourpre foncé et non pas rouge vif, il ne bleuit pas par l'acide sulfurique et n'est pas soluble dans le chloroforme, ni dans le mélange alcool-éther, le sulfure de carbone et l'acétone. L'ammoniaque est sans action sur ce pigment, tandis que l'acide nitrique le dissout. De plus nous n'avons pas trouvé, chez ce poisson, des formes de passage entre les cellules rouges et les cellules jaunes. Il semble donc que le pigment rouge du Thymallus ne rentre pas dans le groupe des carotines; nous espérons en reprendre l'ćtude lorsque nous aurons le matériel nécessaire.

Bondelle (Coregonus exiguus bondella Fatio). Chez ce poisson, nous n'avons trouvé que quelques rares cellules jaune pâle dans les nageoires pectorales et abdominales. Dans ces dernières, il y avait en outre des cellules d'une constitution spéciale; elles étaient formées d'une zone centrale de mélanine entourée d'une auréole ramifiée de pigment bleu vif ou gris bleu. Ce pigment est vraisemblament aussi une carotinalbumine ${ }^{1}$.

1 Voir: J. Verne. Les pigments dans l'organisme animal. Paris, Doin et Cie, 1926. 
Rotengle (Scardinius erythrophthalmus L). Provenance: Léman. Longueur avant l'expérience: 9 à $10 \mathrm{~cm}$. Avant l'élevage ces poissons présentaient les caractères suivants:

nageoires pectorales, sans coloration rouge, mais pourvues de quelques cellules jaunes dans la région distale;

nageoires abdominales, rouges sur la moitié distale;

nageoire dorsale, sans coloration rouge, mais montrant au microscope des cellules jaunes sur toute son étendue;

nageoire anale, rouge dans sa moitié distale;

nageoire caudale, colorée en rouge dans la partie distale du lobe inférieur et en rouge pâle vers l'extrêmité du lobe supérieur.

$\mathrm{Au}$ bout de 261 jours, les individus du lot-vers n'avaient pas modifié leur coloration, sauf que les plages rouges des nageoires abdominales et anale avaient pâli, légèrement, mais d'une façon appréciable. Après le même laps de temps, les Rotengles du lotGammarus montraient les mêmes régions colorées en rouge mais dans une teinte beaucoup plus vive; de plus, les nageoires pectorales possédaient des cellules rouges dans leurs tiers distal et la nageoire dorsale était légèrement colorée en rouge également dans son tiers distal. Vers la fin du premier mois, ces individus avaient déjà acquis la coloration telle qu'elle était encore après 261 jours.

Le pigment rouge des Rotengles, sur le frais ou en solution chloroformique, bleuit très rapidement par l'acide sulfurique.

Chevaine (Squalius cephalus L). Les individus en expérience mesuraient 10 à $15 \mathrm{~cm}$.; au début la coloration rouge était distribuée comme suit:

nageoires pectorales, pourvues de cellules jaune-orange, sauf sur une bande étroite sur leur bord distal;

nageoires abdominales, colorées en rouge-orange pâle dans leur moitié antérieure;

nageoire anale, de même coloration dans ses deux tiers antérieurs;

nageoire dorsale, quelques cellules orange pâle surtout dans la région basale;

nageoire caudale, quelques rares cellules jaunes.

Après 146 jours, les individus du lot-vers n'avaient subi aucune modification dans leur coloration. Ceux du lot-Gammarus au bout 
de 284 jours ne différaient en rien des Chevaines du premier lot, sauf que les plages colorées des nageoires étaient d'un rouge-orange un peu plus vif et que les cellules de la nageoire caudale, au lieu d'être jaunes, étaient rouge-orange. Les ovaires, les testicules et la graisse était incolores.

Spirlin (Spirlinus bipunctatus L). Ces poissons présentaient au début de l'élevage une petite tache brun-jaune à la base des nageoires pectorales, abdominales et anale, la coloration intéressant plutôt la peau que la nageoire elle-même. Toutes les nageoires montraient au microscope quelques très rares cellules jaunes. Le pigment des taches brun-jaune de la base des nageoires ne bleuit pas par l'acide sulfurique; il semble donc qu'il ne s'agit pas là d'une carotine, bien que ce pigment soit soluble dans le mélange alcool-éther. L'élevage des individus du lot-vers a duré 183 à 185 jours, pour les autres l'alimentation en Gammarus a été poursuivie pendant 284 jours. Après l'expérience on pouvait constater que les Spirlins des deux lots ne différaient en rien de leurs congénères sacrifiés au commencement de l'élevage. Nous nous trouvons donc en présence de Poissons ne pouvant fixer qu'une quantité presque infinitésimale de carotine et possédant un pigment brun-jaune ne bleuissant pas par l'acide sulfurique.

Goujon (Gobio gobio L). Nous avons élevé pendant 38 jours un seul individu de cette espèce dans un aquarium peuplé de Gammarus; mais nous ne l'avons jamais vu manger. A sa mort, qui semble due à l'inanition, l'intestin ne contenait que quelques mucosités verdâtres, mais il hébergeait cependant trois Pomphorhynchus laevis, ce qui laisse supposer pue l'animal avait, à l'état libre ou en captivité, absorbé quelques Gammarus. Les nageoires, sauf la nageoire anale, montraient des cellules à carotine rouge-orange, rondes et très petites. Chez un autre mâle la répartition des cellules pigmentaires était la même.

Vairon (Phoxinus phoxinus L). Avant l'élevage les Vairons que nous avons examinés ( 6 individus adultes des deux sexes) n'étaient pas absolument identiques au point de vue de la répartition du pigment rouge, mais les différences constatées étaient individuelles et non pas sexuelles. Si cet examen avait été fait pendant la 
période de fraye, on aurait certainement pu noter quelques différences d'un sexe à l'autre. Sans entrer dans des détails nous pouvons dire que toutes les nageoires paraissaient incolores à l'œil nu, mais que le microscope y révélaient l'existence de cellules jaunes et de cellules rouges, avec toutes les colorations transitoires entre les unes et les autres. En outre, chez quelques individus les bords de la bouche, la région operculaire et la face ventrale en avant des nageoires abdominales ou de la nageoire anale étaient plus ou moins légèrement colorés en rouge. Cette description sommaire, nous pourrions la répéter pour les Vairons du lot-vers qui ont été soumis au régime des vers de terre hâchés et des Enchytraeus pendant 183 à 185 jours. Le deuxième lot de Vairons a été nourri de Gammarus, en dehors de la période de fraye, pendant 176 et 184 jours. La seule différence un peu importante que nous ayons pu noter entre ces deux lots est que chez les poissons du lot-Gammarus les cellules rouges du pourtour de la bouche et de la face ventrale sont passablement plus abondantes. Si l'élevage avait porté sur des alevins, les différences auraient été assurément beaucoup plus importantes. Les Vairons nous ont permit de faire quelques observations dont nous parlerons plus bas au paragraphe "livrée de noce".

Orie (Idus melanotus Heckel, var. miniatus). Cette variété rouge de l'Ide mélanote doit sa coloration brillante également à une carotine, ainsi que nous l'avons constaté par les réactions habituelles. Nous avons soumis deux Orfes à l'alimentation en Gammarus et deux autres ont été nourris de vers de terre, de Tubifex et d'asticots. Après 117 jours, nous avons noté que chez les quatre individus les régions colorées en rouge (corps au-dessus de la ligne latérale et nageoires) étaient de même étendue, mais elles étaient d'un rouge beaucoup plus vif chez les individus du lot-Gammarus. Chez ces derniers la chair n'était pas saumonée, ni sur le frais, ni après coction. Pendant 9 mois nous avons élevé dans un aquarium à parois sombres et très peu éclairé un Orfe de chacun des lots en les nourrissant d'asticots et de graines de lupin. L'un et l'autre ont pâli de façon très notable, mais l'Orfe du lot-Gammarus est resté toujours reconnaissable à sa coloration plus vive. De cela on peut conclure que le facteur lumière peut, chez certains poissons, jouer un rôle dans la production des carotinoïdes. Pour 
les Orfes, d'ailleurs, la coloration rouge semble devoir se manifesfester en l'absence d'une alimentation contenant ces substances; ces Poissons auraient alors la faculté d'élaborer des carotinoïdes. aux dépens d'aliments n'en possédant point.

Tanche (Tinca tinca L). Provenance: Léman. Avant d'être soumises aux régimes habituelles, ces Tanches présentaient sur les. nageoires et dans la région operculaire une légère teinte jaune, mais les cellules jaunes étaient si pâles qu'il était très difficile de les apercevoir au microscope. Bien que les nageoires fussent à peine colorées en jaune, nous avons pu en extraire par le chloroforme une carotine jaune. Le spectre d'absorption de cette solution chloroformique était différent de celui que nous avons obtenu avec les solutions rouges (de Rotengle, par exemple); comme nous le disions plus haut, nous pensons reprendre l'étude spectroscopique de ces différents pigments.

Après 4 mois, les individus du lot-vers n'avaient subi aucun changements au point de vue de leur pigmentation. Egalement après 4 mois, chez les Tanches du lot-Gammarus les cellules à carotine étaient distribuées comme suit:

nageoires pectorales partout, surtout dans la région basale;

nageoires abdominales, anales et dorsales, cellules jaunes partout;

nageoire caudale, cellules jaunes partout, sauf sur les rayons, où les cellules pigmentaires étaient rouge-orange.

Les Tanches du lot-Gammarus étaient dons plus vivement colorées que celles du lot-vers et sur les rayons de la nageoire caudale les cellules jạunes avaient passé au rouge-orange. La graisse et les glandes génitales étaient incolores; la chair n'était pas saumonée, ni sur le frais, ni après coction.

Loche (Nemachilus barbatula L). Avant d'avoir été soumises. aux régimes habituels, les Loches montraient des cellules jaunes, rondes et petites, sur les nageoires pectorales, principalement vers. la base, sur les nageoires abdominales, où elles étaient peu abondantes, sur les nageoires caudale et anale et sur la nageoire dorsale, surtout dans la région basale. Les barbillons étaient colorés en rouge-orange, par de nombreuses cellules de cette couleur. Ovaire, testicules et graisse incolores.

Après 165 jours, la pigmentation cutanée des individus du lot- 
vers ne s'était pas modifiée. Chez les Loches du lot-Gammarus, après 216 jours, nous avons constaté les changements suivants:

nageoires pectorales, coloration jaune orange; cellules jauneorange partout; celles-ci sont surtout très abondantes dans la région du bord distal et forment en certains points des amas assez étendus;

nagoires abdominales, paraissent incolores à l'œil nu; cellules jaunes partout; cellules jaune-orange abondantes dans la région du bord antérieur;

nageoire anale, incolore à l'œil nu; cellules rouge-orange dans la région du bord antérieur;

nageoire dorsale, incolore à l'œil nu; cellules jaunes partout; cellules rouge-orange dans la région du bord antérieur;

nageoire caudale, colorée en rouge pâle; cellules rouge-orange partout, plus abondantes vers les bords supérieur et inférieur;

barbillons, tous colorés en orange assez vifs;

face dorsale et flanes colorés par des cellules jaune-orange très abondantes;

face ventrale avec cellules jaune-orange très peu abondantes, complètement absentes sur une bande longitudinale médiane.

La graisse et les glandes génitales étaient incolores; la chair l'était aussi et ne se colorait pas par la coction. Il en était de même pour un autre individu nourri pendant six mois avec des Gammarus.

Brochet (Esox lucius L). Nous avons eu entre les mains deux adultes de cette espèce dont les nageoires étaient rouge pâle; l'examen microscopique de celles-ci nous a montré que cette coloration était due à une forte hyperhémie de ces appendices; le traitement par le chloroforme et l'acide sulfurique n'y a pas décelé la présence de carotine. Chez une douzaine d'autres Brochets de grande taille que nous avons examinés, les nageoires étaient incolores.

Chabot (Cottus gobio L). Avant l'élevage ces poissons montraient sur toutes les nageoires, en assez grande abondance, des cellules à carotine jaunes ou orangées, ces dernières étant surtout abondantes sur les deux nageoires dorsales et sur la caudale. Après quatre mois, les individus du lot-vers et ceux du lot-Gammarus ne présentaient entre eux, à l'œil nu, aucune différence. Un accident 
survenu à notre matériel ne nous a pas permis de pousser l'examen plus loin; nous avons pu seulement noter que la chair des individus du lot-Gammarus, comme d'ailleurs celle des autres, n'était pas saumonée et ne roussissait pas par la coction. Cependant plusieurs auteurs, notamment LUNEL ${ }^{1}$, nous apprennent. que la chair des Chabots devient rouge par la cuisson.

Lotte (Lotta lota L). Chez les quelques individus que nous. avons examinés les cellules à carotine étaient distribuées comme. suit:

nageoires pectorales, quelques cellules jaunes, petites, rondes ou peu ramifiées;

nageoires ventrales, sans érythrocytes;

nageoire dorsale, peu de cellules jaunes;

nageoire anale, cellules jaunes assez abondantes, petites, rondes. ou surtout ramifiées;

nageoire caudale, comme la précédente;

région dorsale du corps et flancs, cellules jaunes peu abondantes;

Perche (Perca fluviatilis L). Pour les six individus que nous. avons eus sous les yeux, les cellules à carotine avaient la distribution suivante:

nageoires pectorales, pas d'érythrocytes;

nageoires abdominales, cellules rouge-orange dans les deux tiers antérieurs;

nageoire anale, cellules rouge-orange dans les trois quarts antérieurs;

nageoire prédorsale, quelques cellules rouge-orange, surtout sur les rayons;

nageoire dorsale, quelques cellules jaunes, dans la moitié proximale;

nageoire caudale, cellules rouge-orange abondantes, surtout dans les régions dorsale et ventrale.

Livrée de noce. Chez beaucoup de Poissons d'eau douce la parure de noce, des mâles principalement, est caractérisée surtout

1 G. Lunel. Histoire naturelle des Poissons du bassin du Léman, p. 11. Genève, 1874 . 
par l'apparition de taches et de plages rouges, sur les nageoires et sur diverses régions du corps. Des expériences ont montré que cette pigmentation abondante est en rapport direct avec l'activité du testicule ${ }^{1}$, mais jusqu'à présent on n'a pas établi quelle est l'essence même du phénomène. On a supposé que le phénomène était dû à l'étalement des érythrocytes. Mais nous croyons que la véritable explication peut être trouvée dans les observations que nous avons faites sur le Vairon (Phoxinus phoxinus), espèce chez laquelle la pigmentation rouge de la robe de noce est particulierement brillante.

Chez ce poisson on trouve dans la peau, surtout dans la région ventrale, la région operculaire et les nageoires, des érythrocytes arrondis et de petites dimensions, de dimensions si faibles qu'ils ne conférent pas à ces régions une coloration rouge perceptible à l'œil nu; mais, lorsque le Vairon est mort depuis quelques temps, les régions à érythrocytes acquièrent une coloration rouge plus ou moins intense. On peut provoquer le phénomèrie en incisant la peau de la face ventrale et on verra alors l'incision s'encadrer d'une coloration rouge vif. En examinant au microscope la région incisée, on constatera que les érythrocytes se sont étalés sans perdre en rien de l'intensité de leur coloration. Il est même possible de suivre pas à pas le phénomène, qui se produit en quelques minutes dans le voisinage immédiat de l'incision, mais qui est beaucoup plus lent à quelques distance de celle-ci. Les érythrocytes normaux, qui sont donc sensiblement sphériques ou discoïdaux, mesurent de 0,015 à $0,030 \mathrm{~mm}$. Dans le processus que nous décrivons, ils s'allongent, puis poussent des prolongements de différents côtés; ces prolongement se tronçonnent même en s'éloignant de la cellule, de sorte que celle-ci subit, non pas un simple étalement, mais une véritable désagrégation. Les taches rouges résultant de cette désagrégation atteignent en diamètre $0,1 \mathrm{~mm}$. et plus et elles arrivent même à confluer; en conséquence la peau, qui primitivement montrait au microscope un léger pointillé rouge, devient presque entièrement rouge et prend l'aspect qu'elle revêt chez les Vairons en parure de noce. Il semble donc que l'on soit en droit d'admettre que l'ornementation rouge de celle-ci est produite chez le Vairon, et probablement aussi chez d'autres poissons, par

' 1 Voir: S. Kopec. Contribution to the study of the developpement of the nuptial colour of fish. Comp.-rend. Soc. des Sciences de Varsovie, fasc. I, 1918. 
désagrégation des érythrocytes ayant pour effet ou pour cause la mort de ceux-ci. La formation des organes perlés chez divers Poissons montre qu'au moment de la fraye les téguments sont le siège d'une grande activité et il est tout à fait admissible que cette activité ait un retentissement sur les cellules pigmentaires de la peau.

\section{CONCLUSIONS}

De nos expériences et de nos observations nous pourrons tirer les conclusions qui suivent; celles-ci ne sont évidemment valables d'une façon absolue que pour les Poissons qui les ont fournies; mais il est cependant hors de doute que certaines de nos déductions ont une portée plus générale et qu'elles pourraient s'appliquer à beaucoup d'espèces de poissons.

Chez toutes les espèces de Poissons (Salmo fario, Salmo irideus, Salmo fontinalis, Thymallus thymallus, Coregonus exiguus bondella, Scardinius erythrophthalmus, Squalius cephalus, Spirlinus bipunctatus, Gobio gobio, Phoxinus phoxinus, Idus melanotus, var. miniatus, Tinca tinca, Nemachilus barbatula, Cottus gobio, Lotta lota, Perca fluviatilis) que nous avons examinées, sauf chez le Brochet (Esox lucius), nous avons trouvé des carotines.

Celles-ci ont probablement toujours une origine alimentaire.

Elles proviennent, non seulement des Malacostracés, mais aussi des Entomostracés; cependant certains poissons (Idus melatonus, var. miniatus) semblent avoir la faculté d'élaborer de la carotine (ou de la lycopine?) en l'absence de toute alimentation pouvant fournir ces substances ${ }^{1}$.

La carotine exogène (d'origine alimentaire) peut s'ajouter à la carotine (ou à la lycopine?) endogène (Idus melanotus, var. miniatus).

Certains Poissons, notamment le Spirlinus bipunctatus, ne peuvent fixer qu'une quantité presque infinitésimale de carotine.

Pour une même espèce de Poisson, la carotine se fixe toujours dans les mêmes régions du corps.

Il y a une limite à l'extension des régions rouges, de même qu'il y a une limite à l'intensité de la coloration, limites qui ne peuvent être dépassées, même en prolongeant l'alimentation en Crustacés.

${ }^{1}$ Il en est certainement de même pour le poisson-rouge (Carassius auratus). 
La fixation des carotines dans la peau peut être très rapide (Salmo irideus), 36 heures; alevins de $S$. fario, 5 jours, etc.).

La pigmentation rouge présente, chez les adultes tout au moins ( $S$. fario) une assez grande stabilité et elle se maintient intacte malgré la carence d'aliments contenant des carotines.

Chez le Spirlin (Spirlinus bipunctatus) il existe, en plus de la carotine un pigment brun-jaune ne présentant pas tous les caractères des carotinoïdes.

Chez l'Ombre (Thymallus thymallus) il existe, en plus de la carotine; un pigment rouge foncé d'une nature tout à fait différente.

Chez certains Poissons (Thymallus thymallus, Coregonus exiguus bondella) se trouve, en plus de la carotine, une carotinalbumine (?) bleue.

La carotine qui se dépose dans les érythrocytes est d'abord jaune, puis elle peut passer ensuite au jaune-orange et au rouge vif.

La lumière ne joue aucun rôle dans la fixation des carotine exogènes (Salmo irrideus), mais elle peut avoir une influence sur l'élaboration des carotines (ou de la lycopine?) endogènes (Idus melanotus, var. miniatus).

Les Salmonides seuls (Salmo fario, $S$. irideus, $S$. fontinalis) et peut-être aussi les Cottides (Cottus gobio), peuvent fixer les carotines dans leur musculature (chair saumonée).

Certains muscles ne sont pas susceptibles de se colorer par les. carotines (Salmo fario).

Chez certains Salmonides la graisse des viscères et les ovaires. peuvent fixer les carotines.

La rubéfaction intense des téguments qui caractérise parfois la parure de noce se produit vraisemblablement par l'étalement puis par la désagrégation des érythrocytes.

Les sous-espèces ou les variétés basées sur la présence ou l'absence de pigmentation rouge sont sans valeur. 


\section{$2 \mathrm{BHL}$ Biodiversity Heritage Library}

André,

mile. 1928. "Recherches sur le pigment rouge des Poissons d'eau douce." Revue suisse de zoologie 35, 49-65. https://doi.org/10.5962/bhl.part.117619.

View This Item Online: https://www.biodiversitylibrary.org/item/148556

DOI: https://doi.org/10.5962/bhl.part.117619

Permalink: https://www.biodiversitylibrary.org/partpdf/117619

\section{Holding Institution}

American Museum of Natural History Library

\section{Sponsored by}

BHL-SIL-FEDLINK

\section{Copyright \& Reuse}

Copyright Status: In copyright. Digitized with the permission of the rights holder.

Rights Holder: Muséum d'histoire naturelle - Ville de Genève

This document was created from content at the Biodiversity Heritage Library, the world's largest open access digital library for biodiversity literature and archives. Visit BHL at https://www.biodiversitylibrary.org. 